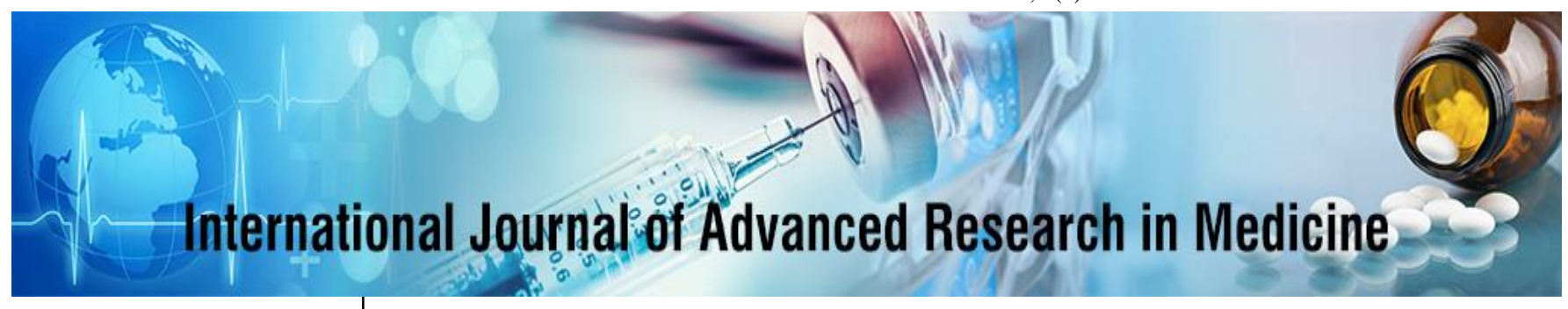

E-ISSN: 2706-9575 P-ISSN: 2706-9567 IJARM 2020; 2(1): 22-25 Received: 21-01-2020 Accepted: 23-02-2020

Dr. Mohan R

Assistant Professor, Department of General Medicine, JJM Medical College, Davangere,

Karnataka, India

Dr. Manjunath Alur Professor and Dean, Department of General Medicine, JJM Medical College, Davangere,

Karnataka, India

Dr. SN Vishwakumar Professor, Department of General Medicine, JJM Medical College, Davangere, Karnataka, India
Corresponding Author: Dr. Manjunath Alur Professor and Dean, Department of General Medicine, JJM Medical College, Davangere, Karnataka, India

\section{A study on microvascular complications in patients with newly detected Diabetes Mellitus}

\author{
Mohan R, Manjunath Alur and SN Vishwakumar
}

DOI: https://doi.org/10.22271/27069567.2020.v2.i1a.34

\begin{abstract}
Introduction: Microvascular complications such as diabetic retinopathy, diabetic neuropathy, and diabetic nephropathy are associated with considerable medical and economic impact among persons with diabetes. In the UK Prospective Diabetes Study [UKPDS], 37\% of patients with newly diagnosed type 2 diabetes developed at least one microvascular complication over a ten years period.

Methodology: The procedure was told to the patients. 5.07 Simmes Weinstein monofilament consisted of a plastic handle supporting a nylon filament. The filament was placed perpendicular to the skin of the foot, and the pressure was applied until the filament buckles. The filament was held in place for approximately 1 second, then released. The patients were asked to elicit a 'Yes/No' response to monofilament pressure and correctly identify the site of contact. Inability to perceive the $10 \mathrm{~g}$ of force it applies was associated with clinically significant large fibre neuropathy. Like this 9 plantar sites and 1 dorsal site were tested in each foot.

Results: Statistical analysis of above obtained data reveals that patient with $\mathrm{HbA} 1 \mathrm{c} \geq 8 \%$ have 4.4 times higher risk of developing microvascular complications than patient with $\mathrm{HbA} 1 \mathrm{c} \leq 8 \%$. This indicates HbA1c is a better predictor of microvascular complications in diabetics. Most of the patients had normal Blood urea and Serum creatinine levels. 25 patients had urea $>40 \mathrm{mg} / \mathrm{dl}$. 2 patient had creatinine $>2 \mathrm{mg} / \mathrm{dl}$.

Conclusion: It is clear that significant increase in Blood urea and Serum creatinine levels which is indicative of renal involvement is less common at diagnosis of diabetes mellitus.
\end{abstract}

Keywords: Micro vascular complications, diabetes mellitus, diabetic retinopathy

\section{Introduction}

Diabetes Mellitus has a become a global health problem due to rapidly increasing prevalence of obesity and physical inactivity. It is common and a serious disease with chronic complications and constitutes a substantial burden for both patient and health care system. There is, therefore, an urgent need to prevent diabetes and its complications ${ }^{[1]}$.

In 2003, the global prevalence of diabetes was estimated at 194 million this figure is predicted to reach 333 million by 2025 as a consequence of longer life expectancy, sedentary life style and changing dietary patterns ${ }^{[2,3]}$.

Diabetes mellitus is characterized by asymptomatic phase between actual onset of hyperglycemia and clinical diagnosis which has been estimated to last at least 4-7 years ${ }^{[4]}$.

Microvascular complications such as diabetic retinopathy, diabetic neuropathy and diabetic nephropathy are associated with considerable medical and economic impact among persons with diabetes. In the UK Prospective Diabetes Study [UKPDS], 37\% of patients with newly diagnosed type 2 diabetes developed atleast one microvascular complication over a 10 year period $^{[5]}$.

Various micro vascular complications in diabetes mellitus includes

- Diabetic Retinopathy

- Diabetic Nephropathy

- Diabetic Neuropathy

With newly diagnosed diabetes mellitus ${ }^{[6]}$. It is seen in $39 \%$ of men and $35 \%$ of women, with marked retinopathy present in $8 \%$ men and $4 \%$ women at the time of diagnosis of diabetes mellitus in UKPDS ${ }^{[7]}$. It remains the leading cause of blindness in working aged Americans. With appropriate medical and ophthalmologic care, more than $90 \%$ visual loss can be prevented. 
The rate of development and progression of retinopathy were significantly reduced after intensive Insulin therapy. $20 \%$ of patients have retinopathy at the time of diagnosis of diabetes ${ }^{[8]}$.

The prevalence of overt nephropathy is about $3 \%$ in newly diagnosed diabetes mellitus ${ }^{[9]}$. Type 1 diabetes with microalbuminuria have median risk ratio of 21 for developing nephropathy and median risk ratio of 8.5 in microalbuminuric type 2 diabetes patients. Diabetes constitutes $44.5 \%$ of end stage renal disease.

Symptomatic peripheral neuropathy is found in up to $1.5 \%$ of newly diagnosed diabetes, whereas by clinical signs it is found in $2.3 \%$ of newly diagnosed diabetes ${ }^{[10]}$. The degree of neuropathy is associated with degree of hyperglycemia [11] and responsible for $50-75 \%$ of non-traumatic amputations.

Presence of microvascular complications at the time of diagnosis of diabetes mellitus are showing increasing trend in India. Early detection of micro vascular complications and its treatment at this time by intensive therapy can prevent progression of these complications and hence morbidity and mortality among patients.

The use of haemoglobin A1C [HbA1C] to diagnose prediabetics and diabetes recently was recommended by American Diabetes Association (ADA) ${ }^{[2]}$. HbA1C testing has an advantage over glucose based testing because it does not require fasting and can be performed anytime. Guidelines recommended an $\mathrm{HbA} 1 \mathrm{C} \geq 6.5 \%$ to diagnose diabetes and $\mathrm{HbA} 1 \mathrm{C}$ between 5.7 and $6.4 \%$ for identifying prediabetes.

Our study is to detect microvascular complications at the time of diagnosis in Indian population.

\section{Methodology}

Sample Size: 100 cases.

Sample procedure: Cross-sectional study.

\section{Clinical Examination \\ Neuropathy}

\section{Peripheral Neuropathy}

Detection of Diabetic Peripheral Neuropathy by:

a) Foot sensitivity testing by Semmes Weinstein mono filament.

b) Deep tendon reflex testing by percussion hammer.

c) Vibration perception testing by $128 \mathrm{HZ}$ tuning fork.

\section{Simmes-Weinstein 10gm monofilament}

Cutaneous pressure perception of the foot was assessed by using $10 \mathrm{gm}$ monofilament. The procedure was explained to the patients. 5.07Simmes Weinstein monofilament consisted of a plastic handle supporting a nylon filament. The filament was placed perpendicular to the skin of the foot, and pressure was applied until the filament buckles. The filament was held in place for approximately 1 second, then released. The patients were asked to elicit a 'Yes/No' response to monofilament pressure and correctly identify the site of contact. Inability to perceive the $10 \mathrm{~g}$ of force it applies was associated with clinically significant large fibre neuropathy. Like this 9 plantar sites and 1dorsal site were tested in each foot.

Insensitivity to $10 \mathrm{gm}$ monofilament at any one site on either foot was taken as abnormal sensation having significant neuropathy.

\section{Autonomic Neuropathy \\ Cardiovascular test \\ Parasympathetic test}

(a) Heart rate response to Valsalva maneuver

The BP falls and heart rate raises during strain phase of Valsalva maneuver and after release BP raises with slowing of heart rate. In patients with autonomic neuropathy BP falls during strain period and after release slowly returns to normal with no change in heart rate. The patient I asked to blow the rubber tubing connected to BP apparatus to raise the pressure to $30 \mathrm{mmh}$ for 15 seconds. The ratio of the length of longest R-R interval and the shortest R-R interval during the manoever is measured. Normal 1.21 or more, abnormal is 1.20 or less.

\section{(b) Heart rate response to deep breathing}

The patient takes deep breaths at the rate of 6 breaths $/ \mathrm{min}$. The maximum and minimum heart rates are evaluated from the ECG recording during each cycle. The difference between three successive breathing cycles gives "maximumminimum heart rate", expressed as E:I ratio.

Normal-15 beats/minute or more. Abnormal- $\leq 10$ beats/minute.

\section{Sympathetic test}

\section{1) BP response to standing up}

$\mathrm{BP}$ recording is done when the subject is lying down and again one min after standing up. The difference in the systolic pressure from lying to standing is taken as a measure of postural hypotension. Normal $10 \mathrm{mmhg}$ or less, borderline-11 to $29 \mathrm{~mm}$ hg and abnormal $-30 \mathrm{~mm} \mathrm{hg}$ or more and diastolic pressure difference of more than $15 \mathrm{mmhg}$ is abnormal.

2) Gustatory sweating

3) Erectile dysfunction

4) Diabetic diarrhea

5) Urinary incontinence

6) Pupillary abnormalities

7) Detection of Diabetic Retinopathy

\section{Results}

Table 1: Showing total number of patients with peripheral neuropathy based on signs and symptoms

\begin{tabular}{|c|c|c|c|}
\hline Peripheral neuropathy & Male & Female & Total \\
\hline Only symptoms & 17 & 12 & 29 \\
\hline Both signs and symptoms & 16 & 12 & 28 \\
\hline Only signs & 5 & 3 & 8 \\
\hline
\end{tabular}

Symptomatic peripheral neuropathy are more common than asymptomatic. $65 \%$ of the patients presented with peripheral neuropathy at the time of diagnosis of diabetes.

$29 \%$ of the patients (17\% males and $12 \%$ females) were presented with only symptoms of peripheral neuropathy, $8 \%$ of them were presented with only signs of peripheral neuropathy, $28 \%$ of them (16\% males and $12 \%$ females) were having both signs and symptoms.

Table 2: Showing number of patients presenting with retinopathy at the time of diagnosis of diabetes mellitus

\begin{tabular}{|c|c|c|c|}
\hline Retinopathy & Male & Female & Total \\
\hline BDR & 9 & 3 & 12 \\
\hline PPDR & 6 & 4 & 10 \\
\hline
\end{tabular}


From the above data it is clear that BDR is more common than PPDR at the time of diagnosis. Totally $22 \%(12 \%$ males and $10 \%$ females) of the patients had retinopathy at the time of diagnosis.

Table 3: Showing number of patients with diabetic nephropathy in patients with newly diagnosed diabetes mellitus

\begin{tabular}{|c|c|c|c|}
\hline Proteinuria & Male & Female & Total \\
\hline Microalbuminuria & 12 & 16 & 28 \\
\hline Macroalbuminuria & 2 & 2 & 4 \\
\hline
\end{tabular}

From the above data it is clear that $28 \%$ of the patients presented with incipient nephropathy at the time of diagnosis. $4 \%$ of the patients had microalbuminuria.

$24 \%$ of the patients were presented with diabetic nephropathy at the time of diagnosis of diabetes mellitus.

Table 4: Correlation of $\mathrm{HbA1C}$ in relation to microvascular complications

\begin{tabular}{|c|c|c|c|}
\hline & Complications & Without complications & \\
\hline HbA1C & $\mathbf{N}(\boldsymbol{\%})$ & $\mathbf{N}(\boldsymbol{\%})$ & Total \\
\hline $6.5-7.5$ & $3(37.5)$ & $5(62.5)$ & 8 \\
\hline $7.6-8.5$ & $12(40)$ & $18(60)$ & 30 \\
\hline $8.6-9.5$ & $18(62.1)$ & $11(37.9)$ & 29 \\
\hline $9.6-10.5$ & $18(81.8)$ & $4(18.2)$ & 22 \\
\hline $10.6-11.5$ & $10(90.9)$ & $1(9.1)$ & 11 \\
\hline Total & 61 & 39 & 100 \\
\hline
\end{tabular}

Statistical analysis of above obtained data reveals that patient with $\mathrm{HbA} 1 \mathrm{c} \geq 8 \%$ have 4.4 times higher risk of developing microvascular complications than patient with $\mathrm{HbA1c} \leq 8 \%$.

This indicates HbA1c is a better predictor of microvascular complications in diabetics.

Table 5: Showing percentage of patients who had lipid in the high risk category at the diagnosis of diabetes

\begin{tabular}{|c|c|}
\hline Lipids (high risk) & Percentage \\
\hline Hypercholesterolemia & $16 \%$ \\
\hline Hypertriglyceridemia & $24 \%$ \\
\hline Decreased HDL cholesterol & $11 \%$ \\
\hline Increased LDL cholesterol & $35 \%$ \\
\hline
\end{tabular}

From the above data it is clear that hyperlipidemia under high risk category was almost equal in both males and females. Total cholesterol, HDL and LDL cholesterol levels were higher in males than females under borderline risk. $24 \%$ of the patients had hyperlipidemia.

Table 6: Showing Blood urea and serum creatinine levels expressed in $\mathrm{mg} / \mathrm{dI}$

\begin{tabular}{|c|c|c|c|}
\hline Blood urea & Male & Female & Total \\
\hline$<20$ & 6 & 10 & 16 \\
\hline $20-40$ & 37 & 22 & 59 \\
\hline$>40$ & 17 & 8 & 25 \\
\hline \multicolumn{3}{|c|}{ Serum creatinine } \\
\hline $1-1-2$ & 23 & 8 & 31 \\
\hline$>2$ & 37 & 30 & 67 \\
\hline 2 & 0 & 2 & 2 \\
\hline
\end{tabular}

Urea: mean $\pm \mathrm{SD}=33.55 \pm 12.35$

Creatinine mean $\pm \mathrm{SD}=1 . \overline{2} 3 \pm 0.48$

Most of the patients had normal Blood urea and Serum creatinine levels. 25 patients had urea $>40 \mathrm{mg} / \mathrm{dl}$. 2 patient had creatinine $>2 \mathrm{mg} / \mathrm{dl}$. From the above data it is clear that significant increase in Blood urea and Serum creatinine levels which is indicative of renal involvement is less common at diagnosis of diabetes mellitus.

\section{Discussion}

In our study $10 \%$ of patients had pneumonia, $4 \%$ had urinary tract infections, $2 \%$ had cellulitis, $2 \%$ had gluteal abscess, 4\% had tubercular pleural effusion. It probably indicates that Indians with diabetes were prone for infections and tuberculosis was little high in Indian study including present when compared to Nambuya AP et al. study.

It is clear that the percentage of both peripheral neuropathy and Autonomic neuropathy was high in our study compared to Ratzmann KP et al. ${ }^{[11]}$ and Parker AL et al. ${ }^{[12]}$ Over all percentage of neuropathy was almost equal when compared to Nambuya AP et al. ${ }^{[13]}$ and high when compared to Thompson TJ et al. ${ }^{[14]}$ and Ramachandran A et al. study ${ }^{[15]}$. Symptomatic neuropathy was present in $36 \%$ of patients in present study and 7\% in Sekar V et al. ${ }^{[16]}$ and $30 \%$ in Tripathi BB et al. ${ }^{[17]}$ study. It was little higher when compared to study by Tripathi $\mathrm{BB}$ et al. and very high compared to V Sekar et al. study.

It is clear that percentage of patients who presented with retinopathy in our study was nearly equal when compared to Rajiv Raman et al. ${ }^{[18]}$ and A. Ramachandran et al. ${ }^{[15]}$ It was more when compared to Thompson TJ et al. ${ }^{[14]}$ and Cathelineau G et al. ${ }^{[19]}$

BDR was the main presenting feature in all the studies including our study and PPDR in our study was almost equal when compared to Wang Y et al. study.

In present study was almost equal to Verinoca R. Collins et al., Weersuriya $\mathrm{N}$ et al. and Cathelineau $\mathrm{G}$ et al. But it was higher than A Ramachandran et al. and Thompson PJ et al. study.

The percentage of microalbuminuria in present study was nearly equal to Cathelineau $\mathrm{G}$ et al. was higher than Ramachandra A et al. study.

The mean FBS and PPBS levels were high in our study than studies done by SS Murthy and Cathelineau G et al. In our study almost all patients had severe fasting hyperglycemia at the diagnosis.

The percentage of patients with hypercholesterolemia was higher in our study than Weersuriya et al. and lower than SS Murthy et al study. The percentage of patients with hypertriglyceridemia was high in our study when compared to Weersuriya $\mathrm{N}$ et al. and less compared to SS Murthy et al. The percentage of patients with decreased HDL was almost equal in present study compared to Weersuriya $\mathrm{N}$ et al.

\section{Conclusion}

- 41 out of 100 newly diagnosed Diabetes Mellitus were presented with microvascular complications.

- Neuropathy was found to be the commonest microvascular complication followed by Diabetic Nephropathy and Diabetic Retinopathy.

- More than 50\% of the patients had Microvascular complications at the time of diagnosis of Diabetes Mellitus.

- HbA1c is a better predictor of microvascular complications in diabetics. 


\section{References}

1. S Barit D, Cooper ME. Mechanism of diabetic nephropathy, Role of Hypertension. 2006; 48:519-26.

2. Wild S, Roglic G, Green A. Global prevalence of diabetes. Estimates for the year 2000 and projections for 2030. Diabetes care. 2004; 24(5):1047-53.

3. Harris MI, Klein R, Welborn TA. Onset of NIDDM occurs atleast 4-7 years before clinical diagnosis. Diabetes care. 1992; 15:815-819.

4. UKPDS Group. Intensive blood glucose contro91 with sulphonylureas or insulin compared with conventional treatment and risk of complications in patients with type II diabetes. Lancet. Erratum in: Lancet. 1998; 352(9131):837-853, 1999; 354(9178):602.

5. Reema M, Deepa R, Mohan V. Prevalence of retinopathy at diagnosis among Type 2 DM patients attending diabetic centre in south India. Br J Ophalmol. 2000; 84:1058-1060.

6. Koher EM, Aldengton SJ, Manley SF, Mathews DR. et al. diabetic retinopathy at diagnosis of NIDDM and associated risk factors. Arch Ophthalmol. 1998; 116:297-303.

7. Elseivier L, Kroneberg HM, Melmed S, Polonsky KS. Williams Textbook of Endocrinology, 15th edn.

8. Droumaguet C. Balkar B, Simon D et al. DESR study group. Use of $\mathrm{HbA} 1 \mathrm{C}$ in predicting progression to diabetes in French men and women: data from an epidemiological study on the insulin resistance syndrome (DESR). Diabetes Care. 2006; 29:1619-1625.

9. Edelman D, Olsen MK, Dudley TK, Harris AC, Odlone EZ. Utility of $\mathrm{HbA1c}$ in predicting diabetes risk. J General Internal Medicine 2004; 19:1175-1180.

10. Pradhan AD, Rifai N, Buring JE, Ridkar PM. HbA1c predicts diabetes but not cardiovascular disease in nondiabetic women. Am J Med. 2007; 120:720-727.

11. Ratzmann KP. Prevalence of peripheral and autonomic neuropathy in newly diagnosed Type-2 diabetics. Journal Diabet. Complications, 1991; 5(1):1-5.

12. Parker AL. Peripheral autonomic impairment in patients newly diagnosed with Type-2 DM. Diabetes care. 1994; 17(12):1422-7.

13. Nambuya AP. The presentation of newly diagnosed diabetic patients in Uganda QJM. 1996; 89(9):705-11.

14. Thompson TJ. Screening of NIDDM in non-pregnant adults. Diabetes Care. 1995; 18:1606-1618.

15. Weersuriya N. Long term complications in newly diagnosed Srilankan patients with type-2 DM. QJM. 1998; 91(6):439-43.

16. Paulsen PL. Epidemiology of microalbuminuria in diabetes and in background Population. Current opinionNephrology Hypertension, 1994; 3(3):248-256.

17. Tripathy BB et al. Diabetic neuropathy. JAPI. 1993; $1: 47$.

18. David JB. Epidemiology of persistent proteinuria in Type-2 Diabetes mellitus, Population based study in Rochester Minnesota. Diabetes. 1998; 37:405-412.

19. Cathelineau G. Management of newly diagnosed NIDDM in the primary setting effect of 2 years of gliclazide treatment. The Diadem study, Metabolism. 1997; 46(12):31-4. 